\title{
Symptomatic and functional outcome of surgical treatment of cervical dystonia
}

\author{
Joachim K Krauss, Elizabeth G Toups, Joseph Jankovic, Robert G Grossman
}

Department of

Neurosurgery, Baylor

College of Medicine,

Houston, Texas

J K Krauss

E G Toups

R G Grossman

Department of Neurology, Baylor College of Medicine, Houston, Texas J Jankovic

Correspondence to: Dr Joachim K Krauss, Department Neurochirurgie, Inselspital, University of Berne, 3010 Berne, Switzerland.

Received 7 January 1997 Accepted 14 April 1997

\begin{abstract}
Objectives-Previous studies of surgical treatment for cervical dystonia have reported highly variable rates of postoperative symptomatic benefit and morbidity. Little is known about functional improvement and long term results. This study evaluates the symptomatic and functional outcome of surgical treatment of cervical dystonia in a consecutive series of 46 patients.

Methods-The most affected muscles were selected for denervation after clinical examination and confirmation by four channel EMG studies. Surgical treatment, aiming at selective elimination of pathological activity while preserving normal motor function and avoiding side effects, was achieved by using a broad scope of techniques including intradural denervation, extradural denervation, and myotomy. Rather than carrying out standard operations, the treatment was tailored to the needs of the patient according to the individual pattern of dystonic activity. Long term benefit was assessed with a global outcome score, and a modified Toronto western spasmodic torticollis rating scale (TWSTRS) in those 34 patients who were available for a recent follow up evaluation.
\end{abstract}

Results-The 46 patients underwent a total of 70 procedures with intradural approaches in 33 instances, extradural approaches in 21 , and muscle sections (singly or combined) in 22 instances. Transient mild postoperative side effects occurred in $10 \%$ of the procedures. The mean duration of long term follow up was 6.5 years. The global outcome was rated as excellent in nine patients $(21 \%)$, as marked in $12(27 \%)$, as moderate in nine $(21 \%)$, as mild in nine $(21 \%)$, and as no improvement in five $(11 \%)$. A persistent side effect consisting of mild difficulty with balance was noted in one case. There were highly significant changes of the preoperative and postoperative mean values for almost all TWSTRS subscores for severity of cervical dystonia, functional disability, and pain. Patients with excellent outcome underwent a higher number of surgical procedures on average than those patients who achieved no benefit. Conclusions-Surgical treatment tailored to the specific pattern of dystonic activity in the individual patient is a valuable alternative in the long term management of cervical dystonia.

\section{(F Neurol Neurosurg Psychiatry 1997;63:642-648)}

Keywords: cervical dystonia; surgical treatment; torticollis

Cervical dystonia is the most common form of focal dystonia. ${ }^{1}$ It is characterised by involuntary, sustained contractions of cervical muscles producing abnormal head movements or postures. The active contractions of the corresponding muscles can be identified by palpation. We prefer the term cervical dystonia rather than spasmodic torticollis as only some patients present with simple turning of the head and the abnormal movement is not always spasmodic. $^{2}$ Furthermore, non-dystonic abnormal neck postures have also been called torticollis. Medical treatment of cervical dystonia often renders limited benefit and side effects are common. ${ }^{34}$ In the past few years, botulinum toxin injections have become the treatment of choice for cervical dystonia. ${ }^{4}$ The injections are effective in most patients and, in general, produce mild side effects. ${ }^{6}$ The effect, however, lasts only three to four months and the injections have to be repeated indefinitely. Botulinum toxin A renders only limited benefit or is not effective in $6 \%-14 \%$ of patients, and it loses its initial efficacy in at least $3 \%$ of patients with continued use. ${ }^{16}$

Surgical treatment of cervical dystonia was considered as early as the last century. ${ }^{7}$ Various procedures have been investigated to achieve permanent relief including functional stereotactic operations such as thalamotomy, spinal cord stimulation, microvascular decompression, myotomy, and denervation techniques. ${ }^{4-12}$ The approaches used most often currently utilise intradural and extradural nerve sectioning techniques. ${ }^{13-16}$ The primary goal of both techniques is to reduce increased muscle tone. Until a few years ago, intradural sectioning of the upper cervical anterior nerve roots (rhizotomy) and the spinal accessory nerve roots was the most common surgical procedure for cervical dystonia. The procedure in general was performed in a standardised manner with bilateral sectioning, not taking into account the specific pattern of dystonic activity in the individual patient. Wide variations in postoperative outcome have been described. ${ }^{15-23}$ Occasionally, severe complications have occurred and some series have reported frequent postoperative morbidity. ${ }^{15} 1824$ Extradural denervation procedures have been favoured more recently. 
With this technique, the posterior primary divisions of the cervical nerve roots (ramisectomy) and the branches of the spinal accessory nerve to the sternocleidomastoid muscle are sectioned. ${ }^{9142526}$ Only the rami and nerves to the dystonic muscles are sectioned, and those not involved in the production of dystonia are spared. Bertrand has coined the term selective peripheral denervation for this approach. ${ }^{9}{ }^{14}$ The use of myotomies has been controversial, although beneficial results have been described in some studies. ${ }^{27}$ Overall, it is most difficult to appreciate the role of different surgical procedures performed for cervical dystonia and to compare their results. The methods of assessment of the outcome in different surgical series have been criticised. ${ }^{4}$ Many studies have used only subjective global outcome scales, and follow up was evaluated by chart reviews or by questionnaires. Little is known about the long term benefit and the improvement of functional disability.

This scenario prompted us to review our experience with the surgical treatment of cervical dystonia over a period of 16 years. We have used and continue to use different surgical options or a combination of techniques such as intradural rhizotomy, peripheral denervation, and myotomy. Instead of performing a standard procedure the technique is adapted to the pattern of dystonic muscle contractions in the individual patient. ${ }^{13}$ The primary rationale for this approach is the selective weakening of the muscles that produce the dystonic posture, with preservation of normal muscle function and the avoidance of side effects. This approach is guided by four channel EMG investigation and may include a subsequent procedure if necessary to produce optimal symptomatic and functional benefit. The present study considers the symptomatic and functional long term outcome as assessed with a modified form of an established clinical rating scale.

\section{Patients and methods}

PATIENT SELECTION AND PREOPERATIVE

ASSESSMENT

Between 1980 and 1996, 52 patients with cervical dystonia underwent surgical treatment at the Department of Neurosurgery at Baylor College of Medicine. Six patients were excluded from this analysis. In three of these patients extradural spinal electrodes were implanted for dorsal column stimulation which, however, proved to be ineffective. The other three patients had thalamotomies performed, but primarily for contralateral appendicular dystonia.

Patients were referred for surgery from the movement disorders clinic. The preoperative routine assessment included extensive neurological examination with videotaped documentation of the movement disorder. Conservative treatment consisted of physical therapy, pharmacotherapy, and, since 1987, botulinum toxin injections. Surgical treatment was considered when the movement disorder was disabling or a cause of considerable social embarrassment, and when it did not respond satisfactorily to drugs, when medical therapy was not tolerated, or when botulinum toxin did not render adequate benefit or became ineffective with repeated injections. Four channel EMG was performed to delineate the involvement of different cervical muscles. Routine recording included the sternocleidomastoid, trapezius, splenius, and semispinalis muscles, and in patients with complex dystonic patterns, the levator scapulae, scalene, and paraspinal erector trunci muscles. Recordings were made at rest and with the head turned to the right and to the left. The degree of the pathological activity was rated semiquantitatively with a five point scale.

\section{SURGICAL PLANNING AND OPERATIVE}

TECHNIQUES

Planning of the surgical procedure was guided by the pattern of dystonic activity in the individual patient. Muscles which did not contribute to the movement disorder were not denervated. Whereas selective intradural techniques were used almost exclusively in the early cases of this series, both intradural and extradural methods were performed in recent patients. Occasionally, the procedure was planned to be performed in successive steps in patients with complex dystonic patterns to avoid excessive postoperative weakness or discomfort. With successive operative steps some patients underwent both intradural procedures and peripheral denervations. Selective muscle sectioning was used only exceptionally as a single operation but commonly as part of stepwise procedures, as an adjunct to another technique, or for treatment of residual dystonia after incomplete denervation. The choice of treatment also depended on the patient's informed decision. Some patients preferred to have a muscle sectioned first rather than an intradural operation with its possible risks, although they were aware that the first might render less benefit. All procedures were performed under general anaesthesia.

The planning strategies may be illustrated best by the following examples. In patient $\mathrm{A}$, with "pure" torticollis to the right, secondary to dystonic activity of the left sternocleidomastoid and the right splenius, alternative procedures would include intradural C1-C3 anterior rhizotomy on the right side and section of the spinal accessory nerve rootlets for the sternocleidomastoid on the left side, or selective peripheral denervation with right sided ramisectomy and peripheral section of the spinal accessory nerve branch to the sternocleidomastoid. If the sternocleidomastoid were to have a very high level of activity and the splenius were to show only very little involvement a possible two step operation could be considered, with the first (and eventually the only) step consisting of myotomy or peripheral denervation of the sternocleidomastoid. In patient $\mathrm{B}$, with torticollis to the right and tilting of the head to the right, secondary to activity mainly of the right splenius, treatment options would include selective intradural C1-C3 anterior rhizotomy on the right side or right sided ramisectomy. In patient $\mathrm{C}$, however, with 
torticollis to the right, tilting of the head to the left, and retrocollis, secondary to bilateral involvement of various muscles including the left sternocleidomastoid, a two step procedure would be performed, with bilateral rhizotomy or ramisectomy as the first step, and peripheral denervation or section of the sternocleidomastoid as the second step. The operative techniques will be outlined briefly.

Intradural section of nerve roots were carried out with the patient in the prone position. Depending on the pattern of the cervical dystonia the procedure involved unilateral rhizotomy, bilateral rhizotomy, or a combination of anterior rhizotomy with contralateral or ipsilateral sectioning of the spinal accessory nerve. Via a posterior midline approach a small suboccipital craniotomy and laminectomies or hemilaminectomies of the upper three cervical vertebrae were performed. After opening of the dura, the anterior $\mathrm{C} 1-\mathrm{C} 3$ roots were identified and sectioned after stimulation. When the procedure was combined with sectioning of the spinal accessory nerve, the accessory nerve was sectioned at the level of the $\mathrm{C} 1$ root.

Extradural section of the posterior rami was done according to the technique described by Bertrand..$^{14}$ Again, depending on the dystonic pattern, either unilateral or bilateral procedures were performed. The dorsal rami were approached via a midline incision and exposed by reflecting the posterior muscles laterally. In general, the dorsal rami were sectioned from C1-C6. The spinal accessory nerve branch to the sternocleidomastoid muscle was sectioned via an incision in the lateral cervical region.

\section{EVALUATION OF OUTCOME}

Recent follow up reviews were sought from all patients who were alive in 1996 . Two of the 46 patients had died secondary to causes not related to the surgical treatment. All patients who could be located were telephoned and were invited for a follow up visit. Standardised telephone interviews were obtained in 16 patients who could not come to Houston, and 18 patients were seen as outpatients. In all these 34 patients a modified form of an established clinical rating scale, the Toronto western spasmodic torticollis rating scale (TWSTRS) ${ }^{28}$ was administered. Additional information was collected from the patients' families.

The severity of cervical dystonia was assessed with graded scales for 12 sub-items. Rotation, head tilt, anteflexion/retroflexion, the percentage of awake time with cervical dystonia, shoulder elevation, range of voluntary motion, and the time the head can be held straight or almost straight, were rated with four point scales; the effect of sensory tricks was rated with a three point scale; and lateral shift, sagittal shift, head tremor, and head jerking were rated with two point scales. The maximum total score for the severity scale was 27 . Functional disability was evaluated with graded scales for 12 subitems including work/domestic work, driving, reading, writing, watching television, outside activities, drinking, eating, personal hygiene, dressing, verbal communication, and the degree of social
Table 1 Patterns of abnormal head postures in 46 patients with cervical dystonia

\begin{tabular}{lc}
\hline & $n(\%)$ \\
\hline $\begin{array}{l}\text { Rotation only } \\
\text { Tilt only }\end{array}$ & $8(17)$ \\
$\begin{array}{l}\text { Rotation and tilt } \\
\quad \text { ipsilateral) }\end{array}$ & $2(4)$ \\
$\begin{array}{l}\text { Rotation and tilt } \\
\quad \text { contralateral) }\end{array}$ & $14(30)$ \\
Rotation and retrocollis & \\
Rotation and anterocollis & $5(11)$ \\
Tilt and retrocollis & $5(11)$ \\
Rotation, tilt, and retrocollis & $1(2)$ \\
& $2(40)$ \\
\end{tabular}

embarrassment. Impairment of the specific function was scored with a six point grading $(0$ $=$ normal, $5=$ extreme or unable to do). The maximum total score for the disability scale was 60. Local pain was assessed with two four point scales, one for severity and one for presence of pain, with a maximum score of 6 .

Preoperative ratings were obtained retrospectively according to the neurological examination before surgery for the severity scale, and according to a questionnaire for the disability scale. The information was verified by review of the videotapes. In addition, global outcome was rated by both the examiner and the patient as: none 0 ; mild 1 ; moderate 2 ; marked 3; and excellent, 4. "Excellent" indicated a total or near total cure of the condition. In patients in whom no recent follow up was obtained, only the global outcome was rated according to the documentation of previous follow up visits. For statistical analysis the paired $t$ test, the Wilcoxon test, the $\chi^{2}$ test, and Fisher's exact test were used.

\section{Results}

DEMOGRAPHIC AND CLINICAL FEATURES

There were 28 women and 18 men. The mean age at the time of the (first) surgery was 46 (range 17-68) years. Cervical dystonia was classified as idiopathic in 35 patients, and as secondary in 11 . There was a history of previous neck trauma in seven patients with idiopathic cervical dystonia. Cervical dystonia was associated with prior exposure to neuroleptic drugs in six patients, meningoencephalitis in two, stroke in two, and it was thought to be secondary to birth injury in one. The average age at onset of cervical dystonia was 36 (range $0.5-65$ ) years. Table 1 shows the distribution of the different patterns of cervical dystonia. Table 2 shows the associations with other movement disorders. All patients had received various drugs before surgery. Limited benefit was achieved with trihexyphenidyl in 18 patients, and with tetrabenazine in 11 . Botulinum toxin injections had been given in 24 patients: five of these patients were

Table 2 Association of cervical dystonia with other movement disorders in 46 patients with cervical dystonia

\begin{tabular}{lc}
\hline & $n(\%)$ \\
\hline Generalised dystonia & $6(13)$ \\
Oromandibular dystonia & $2(4)$ \\
Spasmodic dysphonia & $1(2)$ \\
Segmental dystonia & $1(2)$ \\
Hemidystonia & $1(2)$ \\
Essential tremor & $13(28)$ \\
\hline
\end{tabular}


Table 3 Total number of surgical procedures $(n=70)$ performed in 46 patients with cervical dystonia

\begin{tabular}{lr}
\hline & $n$ \\
\hline Intradural approaches: & 13 \\
Unilateral C1-C3 anterior rhizotomy & 2 \\
Unilateral rhizotomy + ipsilateral SAN rhizotomy & 12 \\
Unilateral rhizotomy + contralateral SAN rhizotomy & 3 \\
Bilateral rhizotomy & 2 \\
Bilateral rhizotomy + unilateral SAN rhizotomy & 1 \\
Unilateral rhizotomy + muscle section & 8 \\
Extradural approaches: & 1 \\
Unilateral ramisectomy & 2 \\
Unilateral ramisectomy + contralateral SAN section & 5 \\
Unilateral SAN section & 4 \\
Bilateral ramisectomy & 1 \\
Unilateral ramisectomy + muscle section & \\
Bilateral ramisectomy + muscle section & 16 \\
Muscle sections: & $(6)$ \\
Muscle sections (only) & \\
Muscle sections (combined - see above) &
\end{tabular}

SAN $=$ spinal accessory nerve.

considered primary non-responders, 14 patients had decreased benefit with repeated injections (usually due to development of immunoresistance), and five had sustained improvement but sought more permanent relief. The mean duration of cervical dystonia at the time of the (first) surgery was 9.8 (range $0.5-53)$ years.

OPERATIVE PROCEDURES AND EARLY

POSTOPERATIVE RESULTS

The 46 patients underwent a total of 70 operations. Table 3 shows the numbers of the different procedures that were used. In 26 patients only one operation was performed, whereas 16 patients had two subsequent operations, and four had three procedures. Second step operations were performed within one month to three years (mean 13 months) after the first procedure. Third step operations were performed within six months to one year after the second procedure.

Different degrees of improvement were seen in all patients in the early postoperative period. In general, patients who underwent denervation procedures noted further benefit within four weeks to six months after surgery. In individual patients cervical dystonia improved gradually for a period as long as four years postoperatively. Transient mild postoperative side effects occurred in seven $(10 \%)$ out of the 70 procedures: a 38 year old woman with tardive dystonia experienced a psychotic episode postoperatively; a 33 year old woman developed cellulitis at the site of the skin incision; one patient had severe headaches; and two patients complained about increased neck pain after intradural approaches; two patients noted difficulty swallowing after bilateral anterior rhizotomies.

LONG TERM RESULTS

The mean duration of postoperative follow up in 44 patients was 6.5 (SD) 4.7 (range $0.5-16$ ) years. No follow up was available in two other patients. The global long term improvement was rated as excellent in nine patients $(21 \%)$, as marked in 12 patients $(27 \%)$, as moderate in nine $(21 \%)$, as mild in nine $(21 \%)$, and as none in five $(11 \%)$. In two of the last five patients cervical dystonia progressed on subsequent follow up. Only one patient with a persistent side effect was identified. This 44 year old man had mild difficulty with balance after intradural denervation. Overall, there was no significant difference in the distribution of outcome scores between patients with idiopathic and secondary cervical dystonia, although only one patient with secondary dystonia had an excellent outcome. There was also no statistical difference in the distribution of outcome when the five patients who primarily did not respond to botulinum toxin injections were compared with the responders.

Table 4 shows the mean preoperative and postoperative subscores for the severity of the cervical dystonia, functional disability, and associated pain according to the modified TWSTRS scale of the subgroup of 34 patients who were available for a recent follow up examination. Highly significant changes were noted for the sum scores and for almost all of the subscores. Of particular note was the change of the rating for social embarrassment. This score ranked highest both preoperatively and postoperatively. It is remarkable that the degree of social embarrassment often did not parallel the degree of functional disability. Some patients with total functional disability in the lower range rated social embarrassment as extreme, whereas other patients with high scores rated it as little or very little. There was no significant improvement in the variables lateral shift, sagittal shift, and head tremor. This may, at least partially, reflect a methodological problem. These variables were rated only with a two point scale-that is, either as being present or absent. Lateral shift, for example, was present in 17 patients preoperatively. The degree of the shift was difficult to quantify.

Table 4 Subscores for the severity of cervical dystonia, functional disability, and associated pain according to the modified TWSTRS scale preoperatively and on long term follow up in 34 patients after EMG guided surgical treatment

\begin{tabular}{lrrr}
\hline & $\begin{array}{c}\text { Preop } \\
\text { Score }\end{array}$ & $\begin{array}{c}\text { Postop } \\
\text { Score }\end{array}$ & P value \\
\hline Total severity score: & 16.35 & 7.88 & $<0.0001$ \\
Rotation & 2.18 & 0.97 & $<0.0001$ \\
Tilt & 1.90 & 0.90 & $<0.0001$ \\
Anteflexion/retroflexion & 1.06 & 0.32 & 0.0001 \\
Percentage of time & 2.82 & 2.00 & $<0.0001$ \\
Shoulder elevation & 1.52 & 0.67 & $<0.0001$ \\
Range of voluntary motion & 1.91 & 0.74 & $<0.0001$ \\
Time head can be held & & & \\
$\quad$ straight & 2.56 & 0.82 & $<0.0001$ \\
Effect of sensory tricks & 1.30 & 0.76 & 0.0004 \\
Lateral shift & 0.39 & 0.27 & 0.125 \\
Sagittal shift & 0.09 & 0.09 & 1.0 \\
Head tremor & 0.41 & 0.32 & 0.37 \\
Head jerking & 0.50 & 0.18 & 0.0068 \\
Total functional disability score: & 34.06 & 14.53 & $<0.0001$ \\
Work/domestic work & 2.91 & 1.53 & $<0.0001$ \\
Driving & 3.15 & 1.62 & $<0.0001$ \\
Reading & 3.42 & 1.65 & $<0.0001$ \\
Writing & 3.06 & 1.46 & $<0.0001$ \\
Watching television & 3.12 & 1.38 & $<0.0001$ \\
Outside activities & 3.44 & 1.56 & $<0.0001$ \\
Drinking & 2.88 & 0.97 & $<0.0001$ \\
Eating & 2.74 & 0.97 & $<0.0001$ \\
Personal hygiene & 2.62 & 0.94 & $<0.0001$ \\
Dressing & 2.41 & 0.77 & $<0.0001$ \\
Verbal communication & 0.79 & 0.35 & 0.0564 \\
Social embarrassment & 3.59 & 1.68 & $<0.0001$ \\
Total pain score: & 4.97 & 2.74 & $<0.0001$ \\
Severity of pain & 2.32 & 1.18 & $<0.0001$ \\
Presence of pain & 2.65 & 1.53 & $<0.0001$ \\
\hline & & & \\
\hline
\end{tabular}


Although it was improved in most of the patients postoperatively, it was abolished only in five patients. The relatively higher $\mathrm{P}$ value for improvement of verbal communication is primarily related to the low sample number, as only nine patients considered their verbal communication as impaired preoperatively. The distribution of the examiner rated global outcome of this subgroup of 34 patients was similar to the distribution of the ratings for the total group of 44 patients, with excellent improvement in nine patients $(26 \%)$, marked in $10(30 \%)$, moderate in $10(30 \%)$, mild in one $(3 \%)$, and none in four $(12 \%)$. There were no differences between examiner rated and patient rated assessments for the extremes of the scale, excellent and no improvement. However, estimations differed, particularly with regard to minor versus moderate improvement. The 34 patients rated their global long term improvement as excellent in nine patients $(26 \%)$, as marked in eight $(23 \%)$, as moderate in seven $(21 \%)$, as mild in six cases $(18 \%)$, and as none in four $(12 \%)$.

Botulinum toxin injections were given to 18 patients in the postoperative course. With progressive benefit of cervical dystonia from the surgical intervention, nine patients abandoned the injections later on. Twenty patients received medical treatment in the further course, but most of them had lower dosages than preoperatively. At the recent follow up, 17 patients did not continue any form of treatment.

When the five patients who did not experience any long term benefit were compared with the nine patients with excellent improvement no differences were noted for preoperative indices such as age at onset, duration of dystonia, type of cervical dystonia, or age at the operation. There was a significant difference, however, with regard to the number of operations performed. Only one of the patients who did not enjoy long term benefit underwent two procedures, whereas nine of the 11 patients with excellent long term outcome had more than one operation $(\mathrm{P}<0.05)$.

\section{Discussion}

The population of this study is comparable with the demographic and clinical data of other series of cervical dystonia as seen in specialised referral centres. ${ }^{2}$ Our study shows that cervical dystonia can be effectively treated with a low rate of side effects by combining several surgical techniques. Whereas excellent or marked outcome was achieved in $48 \%$ of the cases, definite but more limited benefit was evident also in those $42 \%$ of patients with moderate and mild improvement. We think that selective reduction of dystonic activity but preservation of normal function is of crucial importance in surgical treatment of cervical dystonia. Furthermore, for the elective nature of the surgery we think that postoperative morbidity should not be accepted as intrinsic to surgical treatment. Previous studies have shown that the frequency of side effects primarily depends on the extent of the procedure. ${ }^{15}$ Thus, to avoid complications, we consider it advantageous to stage the surgery, if needed. In this case, the patient has to be clearly informed preoperatively that partial improvement after the first surgery is not regarded as a treatment failure but rather an intermediate state from which further improvement can be obtained. The importance of this approach is reflected by the finding that patients with excellent outcome underwent significantly more procedures than patients who did not enjoy long term benefit.

We found preoperative EMG examinations helpful in some cases when the involved muscles could not be adequately identified by examination or palpation. In such cases EMG can be used to assist in tailoring the operative approach. $^{1429}$

The evaluation of functional disability in cervical dystonia is difficult. ${ }^{30}$ This is reflected by the various rating scales that are currently used to assess the efficacy of treatment of cervical dystonia. Many rating scales have methodological problems that need to be further considered. ${ }^{30}$ The TWSTRS has been used mainly for the evaluation of the effect of botulinum toxin treatment. ${ }^{31}$ It has been shown to be both reliable and valid in the assessment of change in severity of disease. So far, surgical series on cervical dystonia have not attempted to quantify the change of functional disability. Judgement on the quality of outcome has been based either on subjective outcome ratings or on single variables only such as head posture, presence of dystonic spasms, or range of head motion. Such approaches are clearly insufficient, given the many side effects in some series. It is questionable whether patients really enjoy postoperative functional benefit when the head is straight, but they have persistent dysphagia and neck weakness. Such issues may also at least partially explain the high variance of outcome in different series which essentially used the same operative technique. For example, by contrast with series on bilateral intradural rhizotomies which reported "success" rates ranging between $70 \%$ and $90 \%$, Hernesniemi and Keränen reported good results in only $9 \%$ of their patients based exclusively on the patients' self assessments for surgical result, disability, and working capacity. ${ }^{19}$

The variable and individualised surgical strategies do not allow comparative analysis of long term efficacy of the different procedures in our study. Furthermore, it is impossible to compare our results with those from other series. As extradural procedures are less invasive with regard to the removal of bone and opening of the CSF space, we have used these techniques more often recently.

Since the development of intradural procedures for treatment of cervical dystonia by Dandy $^{2}$ and by McKenzie ${ }^{33}$ many variations have been described subsequently. The standard techniques include bilateral anterior C1-C3 rhizotomy, unilateral $\mathrm{C} 1-\mathrm{C} 3$ and contralateral C1-C4 rhizotomy, bilateral rhizotomy and unilateral spinal accessory nerve section, and bilateral rhizotomy and bilateral spinal accessory nerve section. The postoperative mortality ranged between $0 \%$ and $2 \%$ in most 
series, ${ }^{15}$ but it was as high as $12 \%$ in single studies. ${ }^{18}$ Moderate to marked postoperative improvement, in general, have been described in $60 \%$ to $90 \%$ of patients, but as outlined above, the proportion of good improvement was reported as low as $9 \%$, occasionally. ${ }^{15-23}$ Colbassani and Wood have estimated that neck weakness may occur in $39 \%$ of patients, dysphagia in about $30 \%$, and shoulder paresis in up to $41 \% .^{15}$ Although, these side effects are transient in many patients, it remains unclear how often they persist. A high variability of other complications has been reported including CSF fistulas, brainstem infarctions, and infections. ${ }^{1524}$ The procedure is safer with the use of microsurgical techniques.

Extradural nerve sectioning techniques have been developed and promoted, chiefly by Bertrand and colleagues. ${ }^{9}{ }^{14}$ By contrast with the standard denervations in series of intradural rhisotomies, Bertrand introduced the principle of selective denervation. Furthermore, with ramisectomy, denervation of laryngeal and pharyngeal muscles in general is avoided. In a series of 260 patients Bertrand described the postoperative outcome as excellent in $40 \%$ of patients, as very good in $48 \%$, as fair in $10 \%$, and as poor in $1 \% .^{14}$ The benefit is thought to be stable in most patients over time. Side effects are rare with this technique and include infection, paraesthesia, pain, and transient dysphagia. Braun and Richter have described a similar, but somewhat different distribution of outcome. ${ }^{25}$ They rated the postoperative benefit as very good in $12 \%$ of their patients, as good in $39 \%$, as moderate in $25 \%$, and as minor or no improvement in $25 \%$. Similar results have also been reported in other series using this technique. ${ }^{26}$ Again, comparison of the results is limited by methodological problems.

Other surgical options are rarely used nowadays for treatment of cervical dystonia. Thalamotomies have been performed successfully, but bilateral lesions increasing the risk of postoperative dysarthria were required in many instances to produce satisfactory relief. ${ }^{10}$ We consider thalamotomy to be indicated in patients who present with both hemidystonia and cervical dystonia. In such cases, thalamotomy occasionally may render excellent improvement of cervical dystonia on long term follow up. ${ }^{34}$ The experience with microvascular decompression of the spinal accessory nerve has been limited, so far. ${ }^{12}{ }^{35}{ }^{37}$ Chen reported excellent or marked improvement in $83.3 \%$ of patients after selective muscle resections. ${ }^{27}$ The advantage of this technique is its comparatively low operative risk. The long term results, however, remain unclear.

For the figures given above for the rate of improvement and the frequency of side effects the question arises whether intradural approaches are still indicated for the treatment of cervical dystonia. Are there any advantages of this technique compared with peripheral denervation? We think that the available data do not allow definite conclusions so far. As shown in our study, selective intradural procedures are not associated with the high complication rates that have been reported for the standard intradural operations. Although commonly not considered as a side effect, patients who undergo ramisectomy have a variable sensory nerve deficit in the area of the greater occipital nerve, which occasionally may be associated with occipital neuralgias. ${ }^{25}$

The indications for surgical treatment of cervical dystonia need to be redefined. By contrast with botulinum toxin injections, surgery can provide permanent relief of this disabling movement disorder. Furthermore, when looking at the postoperative results and complication rates it is necessary to be aware of the fact that about $28 \%$ of patients experience complications such as difficulty in swallowing, neck weakness, and nausea at some time during the course of the treatment with botulinum toxin injections. ${ }^{6}$ Patients with contractures may not improve with botulinum toxin, but may possibly benefit from surgery when denervation is combined with myectomy. There is a need for prospective studies to compare and better define the role of the different treatment options.

We thank Monica L Domingue for assistance in the preparation of the manuscript.

1 Jankovic J, Fahn S. Dystonic disorders. In: Jankovic J, Tolosa E, eds. Parkinson's disease and movement disorders, 2nd ed. E, eds. Parkinson's disease and movement disorders,

2 Jankovic J, Leder S, Warner D, Schwartz K. Cervical dystonia: clinical findings and associated movement disordystonia: clinical findings and associr
ders. Neurology 1991;41:1088-91.

3 Tsui JKC. Cervical dystonia. In: Tsui JKC, Calne DB, eds. Handbook of dystonia. New York: Marcel Dekker, 1995: $115-27$

4 Greene P. Medical and surgical therapy of idiopathic torsion dystonia. In: Kurlan R, ed. Treatment of movement disorders. Philadelphia: Lippincott, 1995:153-81.

5 Jankovic J, Schwartz K. Botulinum toxin injections for cervical dystonia. Neurology 1990;40:277-80

6 Brin MF, Jankovic J, Comella C, Blitzer A, Tsui J, Pullman SL. Treatment of dystonia using botulinum toxin. In: Kurlan $\mathrm{R}$, ed. Treatment of movement disorders. Philadelphia: Lippincott, 1995:183-246.

7 Iskandar BJ, Nashold BS Jr. History of functional neurosurgery. Neurosurg Clin N Am 1995;6:1-25.

gery. Neurosurg Clin N Am 1995;6:1-25.
8 Krauss JK, Grossman RG. Surgical treatment of movement disorders. In: Grossman RG, Loftus CM, eds. Principles of neurosurgery. Philadelphia: Lippincott-Raven, 1997 (in neurosurger

press).

Bertrand CM, Lenz FA. Surgical treatment of dystonias. In: Tsui JKC, Calne DB, eds. Handbook of dystonia. New York: Marcel Dekker, 1995:329-45.

10 Cooper IS. Effects of thalamic lesions upon torticollis. $N$ Engl f Med 1964;270:967-72.

1 Freckmann N, Hagenah R, Herrmann HD, Müller D. Bilateral microsurgical lysis of the spinal accessory nerve roots for treatment of spasmodic torticollis. Acta Neurochir 1986; 83:47-53.

12 Jho HD, Jannetta PJ. Microvascular decompression for spasmodic torticollis. Acta Neurochir 1995;134:21-6.

13 Grossman RG, Hamilton WJ. Surgery for movement disorders. In: Jankovic J, Tolosa E, eds. Parkinson's disease and ders. In: Jankovic J, Tolosa E, eds. Parkinson's disease and
movement disorders, 2nd ed. Baltimore: Williams and movement disorders, 2nd

14 Bertrand CM. Selective peripheral denervation for spasmodic torticollis: surgical technique, results, and observamodic torticollis: surgical technique, results, and
tions in 260 cases. Surg Neurol 1993;40:96-103.

15 Colbassani HJ Jr, Wood JH. Management of spasmodic torticollis. Surg Neurol 1986;25:153-8.

16 Friedman AH, Nashold BS Jr, Sharp R, Caputi F, Arruda J. Treatment of spasmodic torticollis with intradural selective rhizotomies. $\mathcal{F}$ Neurosurg 1993;78:46-53.

17 Gauthier S, Perot P, Bertrand G. Role of surgical anterior rhizotomies in the management of spasmodic torticollis. Adv Neurol 1988;50:633-5.

18 Arseni C, Maretsis M. The surgical treatment of spasmodic torticollis. Neurochirurgia 1971;14:177-80.

19 Hernesniemi J, Keränen T. Long-term outcome after surgery for spasmodic torticollis. Acta Neurochir 1990;103: 128-30.

20 Hamby WB, Schiffer S. Spasmodic torticollis: results after surgical rhizotomy in 50 cases. $\mathcal{F}$ Neurosurg 1969;31:323-6.

21 Hamby WB, Schiffer S. Spasmodic torticollis: results after cervical rhizotomy in 80 cases. Clin Neurosurg 1970;17:2837.

22 Fabinyi G, Dutton J. The surgical treatment of spasmodic torticollis. Aust NZ F Surg 1980;50:155-7. 
23 Speelman JD, van Manen J, Jacz K, van Beusekom GT. The Foerster-Dandy operation for the treatment of spasmodic torticollis. Acta Neurochir Suppl 1987;39:85-7.

Nen faced with a malpractice suit? Clin Neurosurg 1975;23:112-24.

25 Braun V, Richter HP. Selective peripheral denervation for the treatment of spasmodic torticollis. Neurosurgery 1994 35:58-63.

26 Davis DH, Ahlskog JE, Litchy WJ, Root LR. Selective peripheral denervation for torticollis: preliminary results. Mayo Clin Proc 1991;66:365-71.

27 Chen X. Selective resection and denervation of cervical muscles in the treatment of spasmodic torticollis: results in 60 cases. Neurosurgery $1981 ; 8: 680-8$.

28 Consky ES, Lang AE. Clinical assessments of patients with cervical dystonia. In: Jankovic J, Hallett M, eds. Therapy with botulinum toxin. New York: Marcel Dekker, 1994:21137 .

29 Russo LS Jr, Arce C. Simultaneous four-channel electromyography as an adjunct to selective denervation in the treatography as an adjunct to selective denervation in the treat-
ment of spasmodic torticollis [abstract]. Ann Neurol 1990; 28:266.

30 Lindeboom R, De Haan RJ, Aramideh M, Brans JWM, Speelman JD. Treatment outcomes in cervical dystonia: clinimetric study. Mov Disord 1996;11:371-6.
31 Comella CL, Buchman AS, Tanner CM, Brown-Toms NC, Goetz CG. Botulinum toxin injection for spasmodic torticollis: increased magnitude of benefit with electromyographic assistance. Neurology 1992;42:878-82.

32 Dandy WE. An operation for the treatment of spasmodic torticollis. Arch Surg 1930;20:1021-32.

33 McKenzie KG. Intermeningeal division of the spinal accessory and roots of the upper cervical nerves for the treatment of spasmodic torticollis. Surg Gynecol Obstet 1924;39:5-10

34 Krauss JK, Mohadjer M, Braus DF, Wakhloo AK, Nobbe F, Mundinger F. Dystonia following head trauma - a report of nine patients and review of the literature. Mov Disord 1992; 7:263-72.

35 Aksik I. Microneural decompression operations in the treatment of some forms of cranial rhizopathy. Acta Neurochir 1993;125:64-74.

36 Pagni CA, Naddeo M, Faccani G. Spasmodic torticollis due to neurovascular compression of the 11 th nerve. $f$ Neurosurg 1985;63:789-91.

37 Shima F, Fukui M, Matsubara T, Kitamura K. Spasmodic torticollis caused by vascular compression of the spinal accessory root. Surg Neurol 1986;26:431-4. 